\title{
Innovación empresarial y territorio: Una aplicación a Vigo y su área de influencia
}

\section{Carlos María Jardón}

Departamento de Economía Aplicada, Universidad de Vigo, España.

RESUMEN | La innovación es la capacidad de generar e incorporar conocimientos para dar respuestas creativas a los problemas del presente. En la situación actual es vista como un factor clave para mejorar la competitividad de las empresas y favorecer el desarrollo sostenible en los territorios.

Las pymes tienen una mayor dependencia del territorio, por lo que se analiza cuál es el efecto que la gestión del territorio por parte de las empresas tiene sobre su capacidad de innovación. Se evalúa el sistema de innovación de las pymes de Vigo y su área de influencia. A partir de una encuesta entre las pymes de las diferentes cadenas empresariales existentes en la zona y mediante el uso de la técnica de mínimos cuadrados parciales (PLS) se muestra que los factores externos asociados a ese territorio tienen un impacto importante sobre los factores internos asociados a la capacidad de innovación de la empresa.

PALABRAS ClAVE | Desarrollo territorial, economía regional, innovación tecnológica, teoría del desarrollo regional.

ABSTRACT | Innovation is the ability to generate and integrate knowledge to provide creative answers to current problems. In the current situation it is seen as a key factor to improve the competitiveness of companies and to promote sustainable development in territories. SMEs are more dependent to the territory. This essay discuss the effect of land management by companies has on their ability to innovate. It assesses the innovation system of SMEs in Vigo and its surrounding area. From a survey of SMEs in the various existing business clusters in the area and using the technique of partial least squares (PLS) it shows that externalities associated with that territory have a major impact on the internal factors associated the innovativeness of the company.

KEY WORDS | Territorial development, regional economy, technological innovation, regional development theory.

Recibido el 23 de septiembre de 2010, aprobado el 29 de abril de 2011.

Correspondencia: Facultad de Ciencias Económicas y Empresariales, Campus de Lagoas Marcosende, Universidad de Vigo, 36310 Vigo, España. +34-986812491; e-mail: cjardon@uvigo.es 


\section{Introducción}

Las pymes (pequeñas y medianas empresas) competitivas son generadoras de empleo y riqueza en su territorio, potenciando, de esta forma, el desarrollo local. Una empresa es competitiva si sabe situarse en mejor posición que sus competidores en algún aspecto que genere valor para el cliente final (Ireland y Webba, 2007). Una forma de ser competitivas consiste en generar innovaciones, es decir, ser capaz de introducir nuevos productos, nuevas prácticas, nuevas tecnologías o nuevas formas de organización, de manera que produzcan riqueza transfiriendo "los recursos de campos de baja productividad y rendimiento a niveles de mayor productividad y rendimiento" (Drucker, 1988, p. 42).

Hay numerosos factores que hacen que unas empresas sean más innovadoras que otras, sin estar clara la causa de estas diferencias. Una posible respuesta sea la "capacidad innovadora" de las empresas, que indica su habilidad para innovar (Hii y Neely, 2000). Desde los inicios de la teoría del desarrollo endógeno ha sido estudiada la importancia del territorio para mejorar la innovación en las pymes. Dicha innovación está asociada a los Sistemas Nacionales y Regionales de Innovación (Cooke y Morgan, 1998). Estos se apoyan en tres grandes grupos de actores: las empresas, las instituciones de investigación, desarrollo e innovación $(\mathrm{I}+\mathrm{D}+\mathrm{i})$ y las instituciones políticas. Estas últimas gestionan el marco jurídico donde se desarrollan las innovaciones; las segundas gestionan el conocimiento básico y los elementos para elaborar las innovaciones; finalmente, las empresas gestionan el valor que generan las innovaciones, siendo de hecho uno de los agentes que poseen un nivel de importancia superior, ya que generalmente son las que materializan y trasladan los nuevos conocimientos y tecnologías al sistema productivo y a los mercados (CapoVicedo et al., 2007). El territorio ha sido considerado el lugar de encuentro de estos tres grupos de actores y eso permite que se hable de medios innovadores (Méndez, 2002). Es menos habitual analizar el efecto del territorio sobre la innovación desde un enfoque microeconómico, es decir, cómo afecta la organización y utilización de los recursos tangibles e intangibles disponibles para las empresas en un territorio, que le permiten a ésta generar valor para el cliente final. Esto es lo que se denomina "gestión de los recursos territoriales".

Estos hechos nos permiten establecer el objetivo de este trabajo. Nos centramos en las pymes, puesto que ese tipo de empresas tienen una mayor dependencia del territorio. Dentro de éstas analizamos cual es el efecto que la gestión del territorio por parte de las empresas tiene sobre su capacidad de innovación. El territorio es analizado como soporte para los proveedores, la empresa o los clientes y no como fuente de cultura o de mercado laboral, o de legislación, etc..., factores todos ellos de atracción. En ese sentido, podría completarse el modelo teniendo en cuenta esos aspectos complementarios. 
Con esa limitación, el contraste de esta hipótesis se realiza en Vigo y su área de influencia, zona que está situada en el suroeste de Galicia, cerca de Portugal, en la cornisa atlántica de España. Es una comarca con una larga tradición empresarial que se fortaleció a principios del siglo XX con el auge de las conserveras y que fue reforzado a mediados de los años 60 con la implantación de una multinacional del automóvil en la zona. Ha sufrido varias reconversiones industriales que han puesto a prueba su capacidad de recuperación y han demostrado el gran espíritu emprendedor existente. Por estos motivos aparece como un lugar idóneo para contrastar algunas de las teorías elaboradas sobre la innovación territorial.

Para el contraste se realizó una encuesta entre empresarios de la zona. Se definieron una serie de ítems para evaluar tanto la gestión de los recursos territoriales como la capacidad de innovación, preguntándoles cuáles consideraban eran ventajas competitivas en su empresa. Esa valoración subjetiva puede condicionar los resultados, pero es acorde con otros trabajos de investigación (Bontis y Fitz-Enz, 2002). Por otra parte, la necesidad de trabajar con preguntas estratégicas exigió buscar dentro de las empresas a las más profesionalizadas, lo que implicó un sesgo hacia las mayores dentro de este ámbito. Por ello, las conclusiones no se podrían generalizar a microempresas. Además, al ser datos de una época de expansión se puede condicionar la elección de las ventajas competitivas y la situación de la valoración de las empresas. No obstante, al estar todos los factores en igual situación para las empresas y recoger una visión atemporal, los resultados pueden ser generalizables al conjunto de la economía. En ese sentido, sería interesante un seguimiento de la muestra de empresas mediante datos de panel para evaluar la evolución de las variables consideradas.

El esquema del trabajo es el siguiente: En primer lugar, revisamos los elementos del territorio asociados a la innovación que pueden formar parte de la gestión territorial de la empresa. A continuación, estudiamos los elementos que caracterizan el proceso de innovación en la pyme, finalizando con el estudio de la relación entre territorio e innovación. Posteriormente se estudian algunos aspectos sobre Vigo y su área de influencia como zona de contraste. En el apartado siguiente se expone la metodología de trabajo, la realización de encuestas como fuente primaria de información y mediante técnicas PLS se contrastan los ítems componentes de cada constructo, al igual que las relaciones entre ellos. Se finaliza con un apartado de conclusiones.

\section{Territorio e innovación: Marco y fundamentación teórica}

La innovación se define como la capacidad de generar e incorporar conocimientos para dar respuestas creativas a los problemas del presente. En la situación actual es vista como un factor clave para mejorar la competitividad de las empresas y favorecer el desarrollo sostenible en los territorios. Dicho desarrollo no sólo se produce 
en términos de crecimiento económico, sino también desde una perspectiva más integrada. Por ese motivo, los estudios que incluyen entre sus objetivos prioritarios describir e interpretar los procesos de innovación y sus diferentes impactos socioeconómicos se han convertido en una de las líneas de investigación más relevantes de los últimos tiempos en ciencias sociales, con especial protagonismo en el ámbito geográfico de las propuestas teóricas y los estudios empíricos que se realizan desde la geografía económica e industrial (Feldman, 1994).

La innovación es considerada una variable endógena y propia del sistema (Beristain, 2009). Por innovación se entiende la introducción visible de nuevos productos, de nuevos procesos, la creación de nuevos mercados, el diseño de nuevas formas de organización o el empleo de nuevos factores (Rosenberg, 2000). Estas nuevas ideas pueden mejorar la forma en que hasta ahora se están haciendo las cosas, o cambiarlas radicalmente. La innovación puede ser resultado de la investigación de la empresa o la adquisición de nuevas tecnologías o licencias, pero en el caso de las pymes se apoya en su capacidad de innovación. Dicha capacidad (Schumpeter, 1934) tiene en cuenta la capacidad de innovación de procesos, productos y mercados del personal, puesto que permite a los empresarios mantener actualizado el negocio de la empresa (Ireland y Webba, 2007). Otro aspecto asociado es la innovación de mercados se medirá de modo más específico por la capacidad de penetración en nuevos mercados internacionales. Como instrumento necesario de la innovación se incluye la tecnología existente en la empresa (Helfat, 1994). Entre los procesos necesarios para realizar las innovaciones tendremos en cuenta el proceso de diseño (Helfat, 1994) y el sistema de investigación y desarrollo (Teach, 1990).

Por consiguiente, entre los elementos que componen la capacidad de innovación se tienen en cuenta la actitud cultural hacia ella, la tecnología de las empresas y los procesos más directamente asociados a la generación de innovaciones (ver Figura 1). Son muchas las teorías que analizan los factores de innovación. En el Cuadro 1 se recogen algunas de esas teorías y los factores que sugieren. Es interesante observar cómo el territorio aparece como un elemento esencial para la innovación desde diferentes enfoques.

En la capacidad de innovación territorial interviene el concepto de "milieu" que se refiere a un sistema de agentes y elementos económicos, socioculturales, políticos e institucionales, que poseen modos de organización y regulación específicos. El territorio no va a ser sólo el soporte físico de los recursos, de las actividades productivas y de las relaciones económicas y sociales, sino que también va a ser el lugar en el que los agentes se organizan y utilizando sus capacidades y conocimientos producen e intercambian recursos, bienes y servicios (Vázquez-Barquero, 1999). Las pymes regionales se integran en un milieu asociado al territorio en el que desarrollan sus actividades. En esencia, en esta idea se basan los diferentes conceptos de clúster empresarial actualmente considerados (McCann y Arit, 2006). 


\section{CUADRO 1 | Teorías sobre factores que fomentan la innovación en empresas}

\begin{tabular}{|c|c|c|}
\hline ÁMBITO TEÓRICO & FACTORES QUE INFLUYEN EN LA INNOVACIÓN & REFERENCIAS \\
\hline CAMBIO TECNOLÓGICO & $\begin{array}{l}\text { Búsqueda de los incentivos económicos de mercado } \\
\text { La reducción de los costes de producción } \\
\text { Los esfuerzos intencionales de investigación y desarrollo } \\
\text { La acumulación de capital humano } \\
\text { La explotación de nuevas invenciones o } \\
\text { La aplicación de tecnologías maduras a nuevos usos }\end{array}$ & $\begin{array}{l}\text { (Rosenberg, 2000) } \\
\text { (Rosenberg, 2000) } \\
\text { (Teece, 2000) } \\
\text { (Rosenberg, 2000) } \\
\text { (Beristain, 2009) } \\
\text { (Beristain, 2009) }\end{array}$ \\
\hline $\begin{array}{l}\text { VISIONES ECONÓMICAS } \\
\text { DE CORTE } \\
\text { NEOSCHUMPETERIANO }\end{array}$ & $\begin{array}{l}\text { Factores internos a la propia firma y relacionados con su } \\
\text { organización } \\
\text { Innovaciones radicales en empresas pioneras y difusión } \\
\text { al tejido económico } \\
\text { La existencia de recursos humanos cualificados y de } \\
\text { infraestructuras tecnológicas }\end{array}$ & $\begin{array}{l}\text { (Pérez \& Carrillo, 2000) } \\
\text { (Hagerstrand, 1953) } \\
\text { (Méndez, 2002) }\end{array}$ \\
\hline TEORÍA DE LA EMPRESA & $\begin{array}{l}\text { El entorno general en que la empresa opera } \\
\text { El sector económico en el que realiza su actividad } \\
\text { La estrategia competitiva y la disponibilidad de recursos } \\
\text { El tamaño } \\
\text { La implicación de la dirección de la empresa o en el } \\
\text { proceso de innovación } \\
\text { La cultura empresarial innovadora }\end{array}$ & $\begin{array}{l}\text { (Papaconstantinou, 1997) } \\
\text { (Pavitt, 1984) } \\
\text { (Entrialgo et al., 2001) } \\
\text { (Acs \& Audretsch, 1988) } \\
\text { (De Brentani \& Kleinschmidt, } \\
\text { 2004) } \\
\text { (De Brentani \& Kleinschmidt, } \\
\text { 2004) }\end{array}$ \\
\hline $\begin{array}{l}\text { TEORÍA DE RECURSOS Y } \\
\text { CAPACIDADES }\end{array}$ & $\begin{array}{l}\text { Proveedores } \\
\text { Redes colectivas } \\
\text { Cooperación tecnológica } \\
\text { Los recursos naturales } \\
\text { La necesidad de financiación } \\
\text { Estrategias de producción y marketing }\end{array}$ & $\begin{array}{l}\text { (Porter M., 1985; Wagner, 2006) } \\
\text { (Verhees \& Meulenberg, 2004) } \\
\text { (Klofsten \& Scheele, 2003) } \\
\text { (Ricardo, 1817) } \\
\text { (Birley \& Westhead, 1990) } \\
\text { (Zahra \& Das, 1993) }\end{array}$ \\
\hline $\begin{array}{l}\text { TEORÍA DEL DISTRITO } \\
\text { INDUSTRIAL }\end{array}$ & El territorio como núcleo de conexión de empresas & (Sforzi, 1999) \\
\hline $\begin{array}{l}\text { SISTEMAS PRODUCTIVOS } \\
\text { LOCALIZADOS }\end{array}$ & El territorio como localización del sistema productivo & (Benko \& Lipietz, 2000) \\
\hline DESARROLLO LOCAL & El territorio como base del desarrollo local & (Friedman, 1992) \\
\hline $\begin{array}{l}\text { VENTAJAS COMPETITIVAS } \\
\text { DE NACIONES Y REGIONES }\end{array}$ & El territorio como sede de clústeres & (Porter M., 1990) \\
\hline $\begin{array}{l}\text { MEDIO INNOVADOR Y } \\
\text { REDES DE INNOVACIÓN }\end{array}$ & El territorio e innovación en la dinámica productiva & $\begin{array}{l}\text { (Maillat \& Grosjean, 1999; } \\
\text { Vázquez-Barquero, 1990) }\end{array}$ \\
\hline
\end{tabular}

FUENTE ELABORACIÓN PROPIA. 
Las pymes presentan particularidades respecto a la gran empresa. La existencia de una menor complejidad burocrática, una mayor comunicación entre todos los niveles de la empresa y una mayor cercanía al mercado facilitan su innovación, dada su capacidad de respuesta a las necesidades de los clientes (Lloyd-Reason et al., 2002). Entre sus particularidades está la capacidad que tienen de gestionar los recursos y los procesos asociados al territorio para generar innovaciones. Un recurso clave es el capital relacional que se manifiesta en la posibilidad de cooperación con otras empresas e instituciones situadas en el mismo territorio. La gestión de los recursos territoriales incluye los aspectos más cercanos a la zona donde realiza su actividad, que de algún modo pueda generar efectos sobre la innovación. Entre otros se van a tener en cuenta los diferentes eslabones de la cadena de generación de valor (Porter, 1985), puesto que su buena coordinación y el conocimiento de los procesos asociados permiten la innovación organizativa y de procesos de forma directa. Por tanto, consideraremos como variables las zonas de compra, de producción y de venta. Unidos a la zona de compra están aspectos relativos a los proveedores (Wagner, 2006) y los procesos de aprovisionamiento. Junto con la zona de producción se deben tener en cuenta la logística interna y los sistemas de producción y de gestión medioambiental y de la calidad. La zona de venta se asocia a los procesos de distribución o logística externa y de venta (Porter, 1985). Tal como se comentó previamente, entre los recursos físicos de la zona se incluyen los recursos naturales y los financieros y las capacidades asociadas a ellos. El buen uso de los recursos naturales es una potencial fuente de innovación tanto por su forma de utilizarlos como por los productos obtenidos a partir de ellos. Es evidente la necesidad de recursos financieros para el desarrollo de innovaciones. La pyme hace uso de fuentes de financiación fundamentalmente locales.

Por consiguiente, los elementos del territorio que la empresa gestiona se resumen en tres aspectos: los recursos físicos disponibles; los procesos de compra, producción y venta, y como cemento que los unifica, la cooperación existente en las empresas de la zona. La Figura 1 engloba los diferentes componentes de cada uno de esos aspectos.

En todos los casos hay una sospecha de que se podría mejorar la capacidad de innovación de la empresa cuando ésta mejora en alguno de los aspectos indicados en la gestión de los recursos territoriales. Combinando los recursos y capacidades de la empresa con los factores externos, el empresario construye sus competencias distintivas (Grant, 2005), las que le permiten a su pyme ser competitiva. En este caso se han constituido dos competencias: gestión de los recursos territoriales y capacidad de innovación. A partir de los elementos teóricos definidos previamente, establecemos que la gestión de recursos territoriales permite obtener mayor capacidad de innovación de la empresa. El conjunto de las diferentes variables que intervienen en el modelo junto con las relaciones establecidas se recoge de modo gráfico en la Figura 1. 
Figura 1 | Modelo de análisis

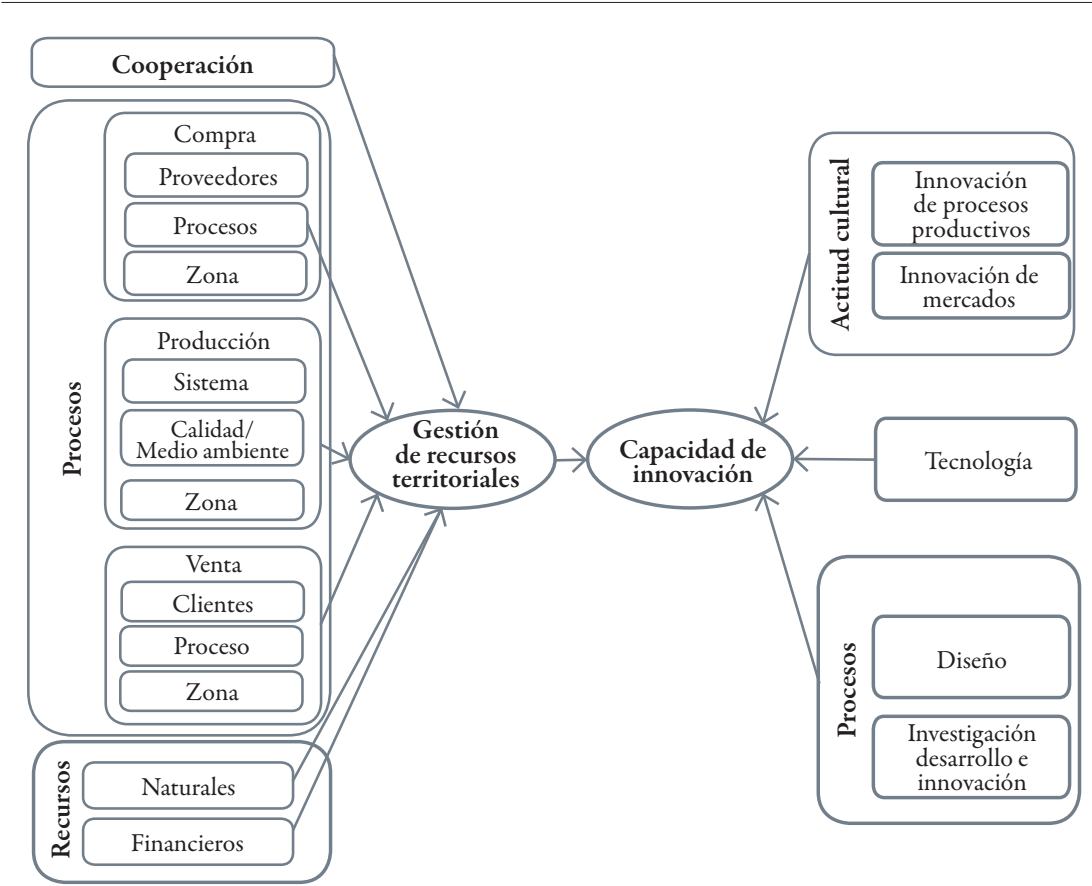

FUENTE ELABORACIÓN PROPIA.

\section{Vigo y su área de influencia}

Galicia es una región NUTS-2 ${ }^{1}$ de la Unión Europea (UE) que se ha mantenido en un crecimiento similar a España. Su peso en la economía española se ha incrementado ligeramente durante los años de la crisis pasando de un 5,1\% en el año 2007 al 5,2\% en el 2010, mientras que la población ha ido disminuyendo en relación a España desde un 6,2 en el año 2007 hasta el 5,9 del año 2010. En el suroeste de la costa gallega, muy cerca de Portugal, se encuentra Vigo. En la actualidad, es la mayor ciudad de Galicia. Dentro del sistema español de ciudades, se puede decir que Vigo se integra, en términos competitivos, en el grupo de ciudades medias portuarias. Comparativamente, cuenta con un perfil industrial más acentuado y un dinamismo económico y demográfico mayor. En el ámbito europeo, Vigo se inserta en el

1 NUTS son las siglas en francés de la Nomenclatura de las Unidades Territoriales Estadísticas utilizadas por la Unión Europea con fines estadísticos. Fueron creadas por la Oficina Europea de Estadística (Eurostat) para dar uniformidad a las estadísticas regionales europeas y son utilizadas, entre otras cosas, para la redistribución regional de los fondos estructurales de la UE. En 1988 fueron aprobadas en la Legislación Comunitaria, pero no fue hasta el 2003 cuando entraron plenamente en función en el reglamento del Parlamento Europeo. La nomenclatura NUTS tiene una estructura jerárquica de tres niveles, de las cuales las regiones NUTS-1 representan la jerarquía mayor, más dos niveles de unidades administrativas locales (en inglés LAU) que también se llaman NUTS niveles 4 y 5. 
arco atlántico del sistema europeo de ciudades a través de su actividad portuaria y empresarial, si bien con una notoriedad e influencia prácticamente limitadas a la Eurorregión Galicia-Norte de Portugal ${ }^{2}$. En el pasado fue uno de los puertos de comunicación con Latinoamérica y aún hoy mantiene lazos familiares y sociales con los países de aquel entorno, si bien los años han hecho que esos lazos fueran desdibujándose.

Como consecuencia de su localización y su carácter de ciudad abierta, cosmopolita, portuaria e industrial, Vigo se situó en el primer tercio del siglo XX a la cabeza del sistema de ciudades de Galicia; a la vez que ocupaba un lugar destacado en el sistema español de ciudades de la época. Posteriormente ha venido experimentando un lento y profundo proceso de transformación, tanto en razón de los propios cambios de su base productiva y social, como de sus funciones urbanas. Todo ello, dentro del marco del proceso de globalización y de formación del nuevo sistema de ciudades y regiones europeas. Sin embargo, los indicadores objetivos de bienestar, tales como la tasa de paro $^{3}$, la sitúan con una cierta desventaja en el sistema regional de ciudades (Provigo, 2002).

Vigo y su área de influencia están formados por 13 municipios, que rodean la ciudad de Vigo y forman el núcleo de la futura área metropolitana de Vigo. La zona en su conjunto tiene 477.768 habitantes, un 6\% del total de Galicia. Está situado en la cornisa atlántica de España con uno de los mayores puertos del país, siendo zona de paso del transporte marítimo entre Europa y África o Latinoamérica. Cuenta con una auténtica "autopista marítima”, con un tráfico de alrededor de 50.000 buques/año que navegan frente a las costas gallegas, pero aún son pocos los que operan en el puerto.

Desde la óptica medioambiental, existe un importante entorno natural, especialmente los espacios naturales y emblemáticos como las Islas Cíes, que forman parte del Parque Nacional de las Islas Atlánticas. Se están llevando a cabo acciones para el saneamiento de la ría de Vigo en particular, y cuidado del entorno metropolitano en general. Cuenta con espacios de elevada calidad ecológica y ambiental, y con un amplio mercado potencial, que facilitan su desarrollo siguiendo estrategias de especialización sectorial y funcional de los municipios del área. Esta es una de sus ventajas competitivas (ver Figura 2).

Es una de las zonas de Galicia que cuenta con un mayor dinamismo económico. Como datos puede observarse en la Figura 3 la evolución de la tasa de creación de empresas de la zona en relación a Galicia, durante los últimos diez años. Incluso en las épocas de crisis dicha tasa se encuentra por encima de la media gallega.

2 En la política europea, una Eurorregión es una forma de estructura para la cooperación transfronteriza entre dos o más países europeos. Las Eurorregiones normalmente no corresponden a ningún gobierno legislativo o institución gubernamental, no tienen poder legislativo propio y sus competencias están frecuentemente limitadas al gobierno local y regional. También son frecuentemente organizadas para promover intereses comunes a través de la frontera y cooperar para el bien común de las poblaciones fronterizas (Consejo de Europa). 
FIGURA 2 | Localización territorial de Vigo y su área de influencia

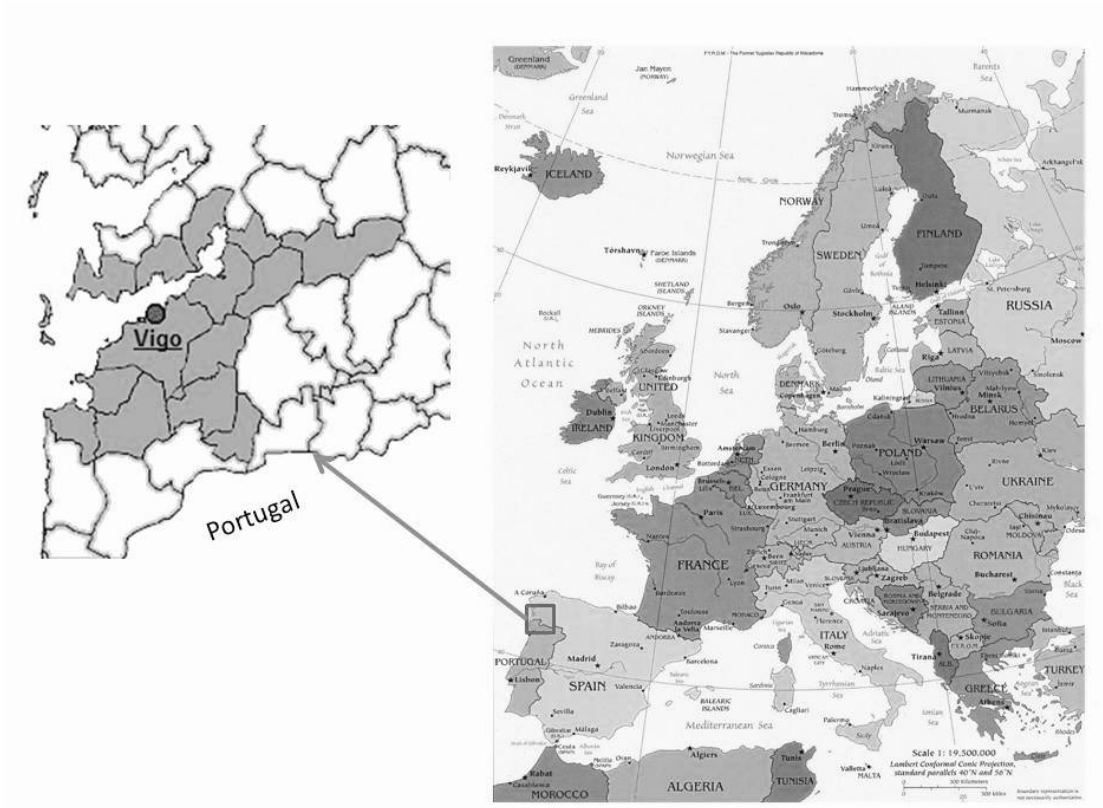

FUENTE ELABORACIÓN PROPIA.

FIGURA 3 | Evolución de la tasa de creación de empresas de Vigo y su área de influencia y Galicia

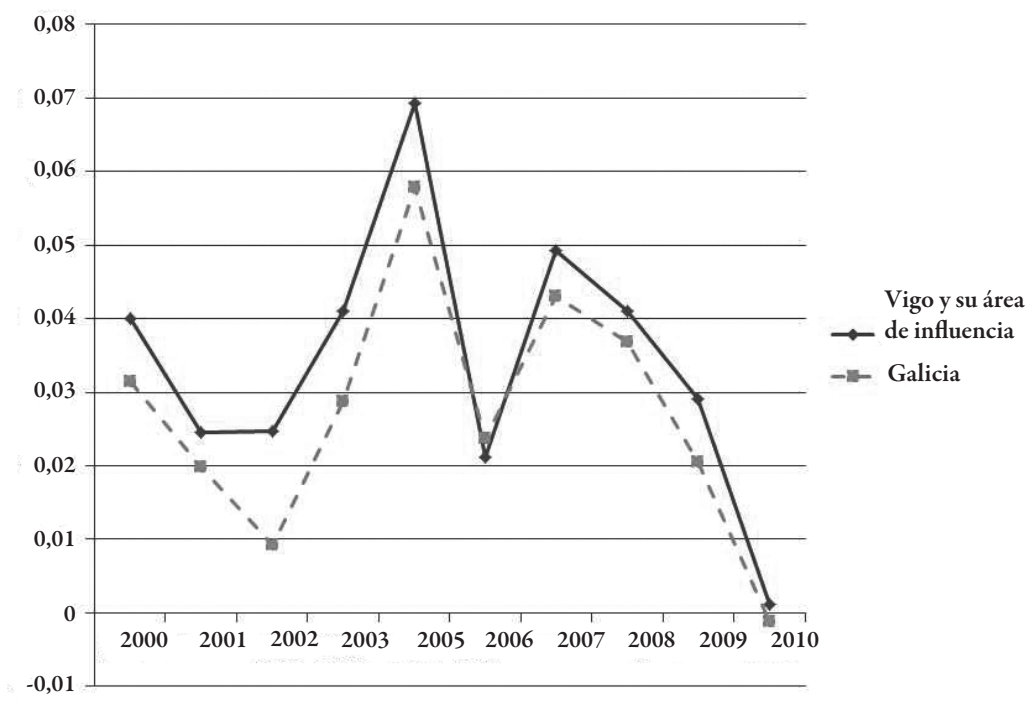

FUENTE ELABORACIÓN PROPIA A PARTIR DE DATOS DE IGE (2010). 
Cuenta con un elevado potencial de desarrollo endógeno disponible, basado en el dinamismo de su sistema productivo. Es un área de carácter metropolitano con una creciente función como centro comercial y de servicios, sobre todo privados, a escala regional. Presenta una posición central en la Eurorregión y una razonable internacionalización económica (ver Cuadro 2). Si bien existen buenas conexiones de transporte por carretera de la ciudad con la meseta y con el centro y norte de Galicia, estas empiezan a estar saturadas, limitando su eficiencia. Además, faltan ferrocarriles eficientes que conecten la ciudad con el exterior.

Vigo y su área de influencia deben, en gran medida, su fuerte crecimiento a la localización del puerto, cuya actividad no sólo no ha decaído, sino que aumenta. Pero este dinamismo incrementa también las tensiones entre una ciudad que pugna por abrirse al mar y un puerto vital para su competitividad y con problemas de espacio para su funcionamiento y expansión. Es un indicador de la importancia económica de la zona. Tiene unos 4,8 km de muelles en dársenas comerciales, 5,93 km de dársenas pesqueras y 3,36 de dársenas para empresas particulares. Está dotado de los principales medios tecnológicos de carga, descarga y almacenamiento tanto fresco como frigorífico. Trabajan 5.140 personas (Autoridad Portuaria de Vigo, 2009). Cuenta con una tradición pesquera a nivel mundial, pero sólo tiene una pequeña participación en el negocio del modo "feeder" de carga y descarga de contenedores, que supone el componente más importante de carga en Vigo ${ }^{4}$. El volumen de tráfico de contenedores sólidos en Vigo ha ido en aumento desde 1980, siendo el segundo más importante del norte peninsular. El Cuadro 2 recoge los datos de la actividad del puerto de Vigo en el año 2009 remarcando la importancia de la actividad nacional e internacional de dicho puerto y de la comarca en su conjunto.

CUADro 2 | Comercio del puerto de Vigo en el año 2009

\begin{tabular}{l|l}
\hline \multicolumn{1}{c|}{ CONCEPTOS } & \multicolumn{1}{c}{ TONELADAS } \\
\hline Comercio exterior & 2.754 .618 \\
Importación & 1.393 .556 \\
Exportación & 1.361 .062 \\
Comercio nacional & 570.573 \\
Transbordadas & 121 \\
Tránsito & 200.659 \\
Pesca fresca, avituallamiento y tráfico interior & 412.811 \\
\hline TOTAL & 3.938 .782 \\
\hline
\end{tabular}

FUENTE (AUTORIDAd PORTUARIA DE Vigo, 2009).

4 Es el transporte de buques portacontenedores, que son los encargados de transportar carga en contenedores estandarizados; se utilizan para transportar todo tipo de mercancías por todo el mundo. Los contenedores son los que se pueden ver habitualmente en los puertos. 
Por su localización geográfica y las condiciones naturales de la ría, tiene excelentes condiciones para llegar a convertirse en un puerto de apoyo de trasvase de carga internacional y de expedición de carga por ferrocarril al resto de España y Europa. Ha sido el motor tradicional de la economía del área, es y seguirá siendo la infraestructura estratégica de internacionalización más relevante de Vigo, sobre la cual cabría organizar una plataforma y comunidad logística que constituya un nuevo factor de competencia de la zona en el escenario europeo e incluso mundial (Provigo, 2002).

\section{El sistema de innovación empresarial}

El sistema productivo de Vigo y su área de influencia se ha caracterizado durante el siglo XX por tener una relevante actitud emprendedora y por ser capaz de dar respuestas eficientes a los cambios del entorno a través de ajustes que promovieron el cambio estructural y su ampliación. Desde inicios de los años noventa se han producido transformaciones significativas en su sistema económico. Su economía sigue estructurada fundamentalmente en torno a la dinámica de las actividades que han sido su motor durante los últimos cincuenta años.

A principios del siglo XX con el auge de las conserveras y la cercanía al puerto se implantan un gran número de empresas asociadas al mar. Posteriormente, a mediados de los años 60 con la implantación de una multinacional del automóvil en la zona se refuerza ese espíritu emprendedor. Estos hechos han significado que las actividades asociadas al mar, tanto la pesca como la alimentación o los astilleros y las que están orientadas al material de transporte, hayan adquirido una gran importancia en la zona. El número de empresas en el año 2009 de la zona era de 73.340 que, frente a las 419.740 existentes en Galicia, corresponden a un 17\% aproximadamente. A partir de las estimaciones del valor añadido bruto (VAB) generado por el sistema productivo empresarial del sector privado realizadas en 2005 (González et al., 2006), y teniendo en cuenta la evolución de la economía gallega en los últimos años (I.G.E., 2009), se estima que esa cantidad corresponde a un 30\% del total de Galicia aproximadamente, mientras que su población es sólo el 6\% del total regional, lo que indica el gran peso empresarial de ese territorio.

Normalmente, al exponer el sistema empresarial de una zona, es conveniente tener en cuenta su estructura, puesto que manifiesta alguna de sus características. Existen diferentes alternativas. En este trabajo se va a seguir un enfoque de cadenas empresariales, es decir, se analizarán de modo conjunto las actividades asociadas a una misma cadena de valor de un producto o servicio (cadena principal) y aquellas otras actividades que sirven de apoyo o son necesarias para la realización del conjunto de actividades anteriores (cadenas de apoyo). Este enfoque permite evaluar las interrelaciones entre empresas y facilitar la constitución de clústeres empresariales. Parece, por consiguiente, una estructura lógica para el estudio de pymes regionales. 
Entre las cadenas principales se incluyen aquellas asociadas directamente a los recursos naturales de la zona, tales como agroindustria, productos derivados del mar y productos derivados de la madera. La cadena de productos relacionados con la piedra trabajada (granito) se incluye dentro de la cadena de construcción y promoción inmobiliaria, puesto que ese producto se orienta casi exclusivamente a esa actividad. Existe también una cadena asociada a productos necesarios para el transporte con gran tradición en la zona, como consecuencia de la instauración de una multinacional de la automoción y de la tradición pesquera que hace que existan diferentes empresas asociadas a la construcción y reparación naval. Otra cadena importante es la textil, moda, confección y complementos que se estudiará asociada al comercio no específico, puesto que éste es el que prima en dichos establecimientos. Esta cadena surge como consecuencia de una tradición existente por el diseño relacionada, en parte, con multinacionales gallegas. Finalmente, entre las cadenas principales asociadas a los servicios cabe estacar la importancia del turismo y las actividades relacionadas con el ocio, dadas las características medioambientales de la zona.

Entre las cadenas laterales y de apoyo se incluyen algunas actividades que suministran material complementario, de equipamiento e instalaciones o dan apoyo a gran número de las cadenas principales, como es el caso de bienes de equipo e industria auxiliar, incluyendo la fabricación de productos químicos, la de logística, transporte y energía - asociada a la distribución- y la de servicios empresariales de apoyo que incluye los servicios financieros y resto de actividades de apoyo. Finalmente, se define una cadena que engloba los servicios sociales que se denomina de educación, salud y medio ambiente. Consecuentemente, se han distinguido once cadenas empresariales para este estudio.

A partir de los datos obtenidos en estudios previos (González et al., 2001;2006) y siguiendo similar metodología se ha estimado la importancia de cada cadena en el VAB y en el número de empresas en la zona de estudio (ver Cuadro 3). Se observa la especialización en productos del mar respecto a las cadenas basadas en recursos naturales. También destaca la importancia del material de transporte y de bienes de equipo. La diferencia entre empresas y generación de VAB indica los sectores con grandes empresas, destacando entre todos ellos la cadena de material de transporte.

Un factor decisivo en la futura transformación del sistema productivo y su ajuste a las condiciones de la competencia existente en los mercados es la capacidad de las empresas para introducir innovaciones y la disponibilidad de recursos humanos cualificados. En un mundo cada vez más integrado económicamente, la competitividad de las empresas aumenta a medida que el conocimiento se incorpora en los productos y bienes a través de la difusión de las innovaciones y de la cualificación de los recursos humanos. El mantenimiento y mejora a largo plazo del sistema productivo local demanda profundizar en su proceso de modernización, expansión y diversificación; en especial, por la vía del incremento de su internacionalización y la mayor introducción de innovaciones a los fines de su aprovechamiento por las empresas. Ahí entran en juego las instituciones de $\mathrm{I}+\mathrm{D}+\mathrm{i}$ de la zona. 
CUADRO 3 | Porcentaje de VAB y empresas de Vigo y su área de influencia por cadenas empresariales

\begin{tabular}{|c|c|c|}
\hline CADENA EMPRESARIAL & $\%$ VAB & \%EMPRESAS \\
\hline Pesca y Productos Transformados del Mar & $10,63 \%$ & $6,42 \%$ \\
\hline Forestal y Productos Transformados de la Madera & $2,57 \%$ & $4,74 \%$ \\
\hline Agricultura y Ganadería y sus Transformados & $2,17 \%$ & $4,26 \%$ \\
\hline Construcción y Promoción Inmobiliaria & $13,86 \%$ & $18,69 \%$ \\
\hline Material de transporte & $14,50 \%$ & $2,95 \%$ \\
\hline Bienes de Equipo, Industria Auxiliar y Productos Metálicos & $11,31 \%$ & $8,43 \%$ \\
\hline Textil-Confección-Moda y Complementos & $7,99 \%$ & $11,15 \%$ \\
\hline Turismo, Ocio y Relacionados & $8,79 \%$ & $14,29 \%$ \\
\hline Educación, Salud y Medio ambiente & $5,40 \%$ & $6,22 \%$ \\
\hline Servicios de apoyo & $14,08 \%$ & $14,86 \%$ \\
\hline Servicios de logística y energía & $8,71 \%$ & $8,00 \%$ \\
\hline
\end{tabular}

FUENTE ELABORACIÓN PROPIA A PARTIR DE GONZÁLEZ ET AL. (2006)

Entre éstas se encuentran las universidades y organismos públicos de investigación, como generadoras del conocimiento básico y las organizaciones y estructuras de apoyo a la investigación y la innovación que conectan dicho conocimiento con las empresas.

Dentro del primer grupo existen en la zona un centro universitario, un centro del consejo superior de investigaciones científicas y uno del Instituto Oceanográfico español. Todos ellos tienen una buena capacidad científica y tecnológica (UVigo, 2009). Los otros dos organismos más orientados a la investigación también han participado en gran número de proyectos nacionales e internacionales publicando numerosos artículos en revistas de prestigio (IIM, 2009; IEO, 2009) 5 . Sin embargo, todavía falta una mayor integración entre estas investigaciones y la innovación empresarial. Una de las causas es el hecho de que el sistema universitario no prevé una armonización entre estas instituciones y el sistema productivo, exigiéndose un notable esfuerzo para completar el ciclo de investigación/mercado, que ofrece a la venta sus resultados de investigación y dificultando transformar esas ideas en nuevas

5 En particular, la Universidad de Vigo durante el año 2007 consiguió más de 25 millones de euros en recursos de $\mathrm{I}+\mathrm{D}+\mathrm{i}$ mediante contratos y proyectos, se han propuesto cinco nuevas patentes y, según datos de su memoria, se han publicado 2.611 artículos de investigación, ha participado en 722 proyectos nacionales e internacionales y ha realizado 214 contratos de investigación aparte de cursos e informes. 
empresas (spin-offs $\left.{ }^{6}\right)$. Existen grupos de excelencia en dichos centros, pero hay dificultades para transformar los resultados de innovación debido a la estructura actual de la universidad que potencia un tipo de investigación no suficientemente utilizada por el tejido productivo. Sin embargo, ese gap va disminuyendo, puesto que sus áreas de especialización son muy cercanas a los sectores productivos de la zona.

El sistema de salud (CHUVI, 2009) tiene unas características específicas. Está basado en el conocimiento, con áreas de alta tecnología (farmacia, instrumentación científica) y campos de cultivo de la innovación, ya que dan apoyo a las necesidades en aspectos diagnósticos y terapéuticos. Los centros de atención de la salud combinan la capacidad de investigación focalizada, la investigación básica, tanto clínica como epidemiología, los resultados sanitarios y la investigación en tecnologías (en colaboración con la industria farmacéutica, el desarrollo de nuevos instrumentos científicos, etc.) en el mismo entorno. Es a menudo dentro de dichos centros donde se produce la generación de conocimiento y su aplicación, con la innovación en los procesos de atención como resultado ${ }^{7}$.

El sistema de organizaciones y estructuras de apoyo a la investigación y la innovación está formado por cinco centros tecnológicos, laboratorios de ensayo y medición, un parque tecnológico, una oficina de transferencia de tecnología (OTRI) y otros organismos que prestan apoyo a la oferta y difusión de tecnología y a la innovación.

Los centros tecnológicos son agentes importantes para la colaboración con grupos de investigación y empresas en la innovación y las mejoras en los protocolos de pruebas y puesta a disposición de los equipos de investigación de bases de datos para la realización de determinados proyectos. Tienen diferentes acreditaciones y certificaciones que les permiten dar apoyo a las empresas de la zona ${ }^{8}$. Además, conocen muy bien las necesidades de las empresas y, en general, colaboran de modo directo con ellas (Retgalia, 2010). Se está estableciendo un parque tecnológico en el que actualmente ya están radicados dos de los centros tecnológicos mencionados previamente, si bien aún se encuentran en su fase inicial. Al estar situado muy cerca del campus universitario constituye un espacio físico de encuentro entre el sector público y la identificación del tejido privado y por lo tanto una herramienta esencial para el enraizamiento y la generación de $\mathrm{I}+\mathrm{D}+\mathrm{i}$. Otros organismos de apoyo a la financiación de la innovación han adoptado estrategias de especialización en ciertos nichos, pero su actividad va más allá de todo el sistema. Las organizaciones dedicadas a la transferencia de tecnología y el conocimiento tienen asignadas las funciones administrativas necesarias para completar la promoción y comercialización de tecnologías?.

6 En el año 2007 se crearon tres spin-off en la Universidad de Vigo.

7 Los investigadores del Consorcio Hospitalario Universitario de Vigo, que engloba todos los centros de salud públicos de Vigo, han publicado cerca de 100 artículos de investigación en el año 2008 y están participando en más de 30 proyectos de investigación, además de atender todas las consultas sanitarias del entorno.

8 Entre ellos trabajan alrededor de 900 personas, de las cuales un 6\% son doctores. Durante los últimos años han realizado más de 200 proyectos de investigación aplicada, se han presentado cinco marcas registradas y cinco patentes (Retgalia, 2010).

9 Estas oficinas gestionaron más de 200 contratos de $\mathrm{I}+\mathrm{D}+\mathrm{i}$, más de 600 informes y alrededor de 50 cursos durante el año 2008 (UVigo, 2009). 


\section{Análisis empírico: datos, modelo seleccionado y resultados}

Dentro de este ámbito espacial nos centraremos en analizar cómo la gestión de este entorno territorial afecta a la capacidad innovadora de las pymes viguesas. El objetivo de la hipótesis propuesta consiste en comprobar si los recursos territoriales pueden ser considerados como factor de innovación. Por lo tanto, nos interesa saber si la combinación de los recursos y capacidades de la empresa asociados al territorio promueven la capacidad de innovación. De acuerdo con ese objetivo haremos uso de la técnica de mínimos cuadrados parciales (PLS) para evaluar el modelo completo.

Todo este proceso lleva consigo varios elementos de trabajo. Por una parte, debemos determinar la población de estudio y la muestra sobre las que se van a contrastar las hipótesis. Se deben discutir las medidas de las variables utilizadas y los constructos definidos. Después, comentaremos la metodología estadística utilizada para los contrastes, para terminar con el análisis empírico de los resultados.

\section{La población y la muestra}

Dado que la estructura empresarial de una zona manifiesta alguna de las características de ésta, convenía diseñar la muestra teniendo en cuenta dicha estructura. En este trabajo se sigue un enfoque de cadenas empresariales, tal como se comentó previamente. Considerando la situación específica de este estudio para facilitar la representatividad, por cada cadena se han distinguido once cadenas empresariales. La importancia de cada cadena en la zona era diferente según se considerara su generación de VAB o el número de empresas existente en la actividad. Para tener en cuenta los dos aspectos se establece un diseño muestral en el que se combinan ambos.

Para obtener la información empírica se llevó a cabo una encuesta en esa zona en el año 2005 entre pymes ${ }^{10}$ de Vigo y su área de influencia. Inicialmente se seleccionó una muestra aleatoria proporcional de 400 empresas, que con un nivel de confianza del 95,5\% nos daría un error máximo en el caso de una pregunta dicotómica del $5 \%$ en todo el conjunto. Se buscó que hubiera un mínimo número de empresas en cada cadena empresarial, para asegurar la representatividad de la muestra. Por ese motivo se seleccionaron aleatoriamente veinte empresas en cada cadena y el resto de la muestra se repartió proporcionalmente y de modo aleatorio de acuerdo a la estructura combinada. Se contactó con el empresario para concretar fechas de envío y de recogida. Una vez acordado, se le envió la encuesta y se pasó a recogerla en la fecha prevista. En caso de duda sobre la encuesta se le asesoró para completar su contenido. La tasa de respuesta fue del $59 \%$, por lo que finalmente se obtuvieron 236 respuestas válidas con las que se elaboró el estudio. Se comprobó que la estructura final de la muestra se ajustaba la población de estudio mediante un test de homogeneidad de poblaciones (Newbold et al., 2002).

10 Se entiende por "pyme" a efectos de la encuesta aquellas empresas con más de 10 trabajadores y menos de 250, que tuvieron una facturación mayor de 50 millones de euros. De ese modo se asegura una mayor tasa de respuesta de la encuesta dado su alto contenido estratégico y no se afecta de modo importante a la representatividad de los resultados considerados en términos de producción. 


\section{Medidas y validez}

Para definir las variables y constructos que intervienen en el modelo se hizo uso de diferentes ítems que aproximaban su valor.

\section{CUADRO 4 | Ítems de la encuesta}

\begin{tabular}{|c|c|c|}
\hline CONSTRUCTO & ÍTEMS CONSIDERADOS & $\begin{array}{l}\text { REFERENCIAS } \\
\text { BIBLIOGRÁFICAS }\end{array}$ \\
\hline $\begin{array}{l}\text { GestióN DE } \\
\text { LA ZONA }\end{array}$ & $\begin{array}{l}\text { El proceso de aprovisionamiento } \\
\text { El sistema de producción/logística/almacenamiento/ } \\
\text { distribución } \\
\text { La actitud de cooperación y alianzas por parte de la } \\
\text { empresa (con otras empresas, con la AA.PP, con el entorno) } \\
\text { El tipo de proveedores } \\
\text { El proceso de comercialización y venta } \\
\text { Las áreas geográficas en las que compra } \\
\text { Las áreas geográficas en las que produce } \\
\text { Las áreas geográficas en las que vende } \\
\text { Los recursos financieros/estructura económico financiera } \\
\text { Los sistema de calidad/gestión medioambiental. } \\
\text { Los recursos naturales }\end{array}$ & $\begin{array}{l}\text { (Porter M. , 1985) } \\
\text { (Porter M. , 1985) (Zahra \& } \\
\text { Das, 1993) } \\
\text { (Klofsten \& Scheele, 2003) } \\
\text { (Cal et al., 2007) } \\
\text { (Fukugawa, 2006; Wagner, } \\
\text { 2006) } \\
\text { (Porter M., 1985) } \\
\text { (Porter, 1990; Vázquez- } \\
\text { Barquero, 1999) } \\
\text { (Porter, 1990; Vázquez- } \\
\text { Barquero, 1999) } \\
\text { (Porter, 1990; Vázquez- } \\
\text { Barquero, 1999) } \\
\text { (Balakrishnan \& Fox, 1993) } \\
\text { (Kim \& Kogut, 1996) } \\
\text { (Porter, 1985; Vázquez- } \\
\text { Barquero, 1999) } \\
\text { (Balakrishnan \& Fox, 1993) } \\
\text { (McLaran \& McGowan, } \\
\text { 1999) }\end{array}$ \\
\hline $\begin{array}{l}\text { CAPACIDAD DE } \\
\text { INNOVACIÓN }\end{array}$ & $\begin{array}{l}\text { El proceso de diseño } \\
\text { El proceso de investigación/desarrollo/innovación } \\
\text { Las tecnologías de procesos y productos utilizadas } \\
\text { La capacidad de innovación de procesos, productos o } \\
\text { mercados } \\
\text { Capacidad de penetración en nuevos mercados } \\
\text { internacionales }\end{array}$ & $\begin{array}{l}\text { (Helfat, 1994) } \\
\text { (Teach, 1990) } \\
\text { (Kim \& Kogut, 1996; Helfat, } \\
\text { 1994) } \\
\text { (Rodeiro \& López, 2007) } \\
\text { (Cal et al., 2007) }\end{array}$ \\
\hline
\end{tabular}

FUENTE ELABORACIÓN PROPIA. 
La forma del cuestionario siguió el esquema de diferentes trabajos (González et al., 2001) basados en escalas contrastadas en la literatura (Narver y Slater, 1990; Malhotra, 1981; Deshpande y Golhar, 1994). En cada uno de los ítemes se les pedía que valoraran si el aspecto que se indicaba en el Cuadro 4 era importante como ventaja competitiva de su empresa, en una escala que iba desde 1 (no es importante como ventaja competitiva) hasta llegar al valor 5 (es muy importante como ventaja competitiva). Este tipo de valoración permite discriminar entre cada uno de los ítemes considerados.

\section{Técnicas estadísticas}

El modelo que se ha planteado intenta evaluar los efectos entre variables estructurales. La forma más sencilla de hacerlo es mediante el uso de relaciones lineales. Existen diferentes técnicas para afrontar este problema. Las más conocidas son el análisis basado en covarianzas (Hair et al., 2006) o la estimación directa de las relaciones estructurales mediante técnicas de mínimos cuadrados (Chin, 1998). Las soluciones basadas en la covarianza demandan unas suposiciones muy exigentes, especialmente el comportamiento normal conjunto de las variables utilizadas en el modelo. El hecho de trabajar con datos ordinales medidos en una escala de intervalo, al estar basados en encuestas según la escala de Likert, hace difícil que se verifique la suposición de normalidad de las variables. Por ese motivo, se ha hecho uso de la técnica PLS que es más flexible y menos condicionada por esas suposiciones. Las soluciones basadas en PLS intentan minimizar la varianza de todas las variables dependientes. Además evita problemas de identificación de parámetros como ocurre en los modelos de covarianza.

Dicha técnica utiliza como criterios de validación la varianza promedio extraída (AVE), el alfa de Cronbach (Cronbach, 1951) y la fiabilidad compuesta. Se recomienda que AVE sea mayor que $50 \%$ para que los indicadores expliquen más que el error, pero si ese valor se encuentra alrededor de 0,3 se puede aceptar también si está muy justificado. Las otras dos miden la consistencia interna del cuestionario. Producen valores que oscilan entre cero (0) y uno (1). Mientras más cerca del valor uno (1), más confiable es el instrumento. Los criterios que utilizamos para la interpretación de ambos coeficientes son los valores propuestos por Nunnelly (1978): menores de 0,6 (baja); entre 0,61 y 0,70 (adecuada); entre 0,71 a 0,80 (buena); mayores de 0,80 (alta).

$\mathrm{Al}$ no exigir la normalidad de las variables que definen los constructos a la hora de hacer la estimación, la ley de distribución de los estimadores obtenidos no es conocida. Por ello, para contrastar si los parámetros son significativos se hace uso de una técnica de Bootstraping. Ésta consiste en crear $\mathrm{N}$ muestras similares a la obtenida con esa misma distribución empírica. A partir de cada una de ellas se obtendrá un estimador PLS diferente. Suponiendo que el promedio de todos ellos sigue aproximadamente una distribución normal se evalúa su fiabilidad y valor (Efron, 1979). 
Para el proceso de análisis, depuración y tratamiento de los datos, determinación de factores y evaluación de efectos se utilizó el programa Statistical Package for the Social Sciences (SPSS versión 15) y Visual Partial Least Squares (VPLS 1.4).

\section{Análisis empírico de los resultados}

Dado el esquema de trabajo propuesto consideramos dos grandes aspectos que englobaremos cada uno de ellos en un constructo predeterminado. Los hemos denominado respectivamente "gestión de recursos territoriales" (ZONE) e "innovación empresarial" (INNO). Cada uno de ellos ha sido definido con las variables previamente enunciadas. El modelo ha sido estimado de modo global mediante la técnica PLS (Chin, 1998).

Esta técnica presenta los resultados en tres aspectos complementarios. En el primero se estudia la representatividad de los constructos; en el segundo se analizan las ponderaciones de cada variable original sobre cada uno de los constructos predefinidos y finalmente se estiman los parámetros que las relacionan.

La representatividad de los constructos viene medida por la fiabilidad del modelo. Los resultados de dichas medidas muestran características diferentes según los datos analizados (ver Cuadro 5). La fiabilidad compuesta y el alfa de Cronbach indican en todos los casos una coherencia suficiente.

\section{CUADro 5 | Medidas de fiabilidad}

\begin{tabular}{c|c|c|c}
\hline CONSTRUCT & COMPOSITE RELIABILITY & AVE & CRONBACH ALPHA \\
\hline Zone & 0.881090 & 0.383963 & 0.850363 \\
Inno & 0.852678 & 0.538569 & 0.773872 \\
\hline
\end{tabular}

FUENTE ELABORACIÓN PROPIA.

El Cuadro 6 recoge la ponderación de cada uno de los ítemes en los constructos definidos. En todos los casos se observa que se pueden considerar sus efectos significativos utilizando la técnica bootstrap. Dicho cuadro se ha dividido en dos partes: La primera incluye las doce primeras filas después del encabezado, mientras que la segunda contiene el resto. Esa primera parte se refiere a la gestión de los recursos territoriales y está asociada a los recursos tangibles y los procesos relacionados con el territorio. 
CUADRO 6 | Medidas de las cargas factoriales y sus coeficientes bootstrap

\begin{tabular}{|c|c|c|c|c|c|}
\hline & & ENTIRE & MEAN & STANDARD & T-STATISTIC \\
\hline & & SAMPLE & OF & ERROR & \\
\hline & & ESTIMATE & SUBSAMPLES & & \\
\hline \multirow[t]{12}{*}{ ZONE } & $\begin{array}{l}\text { La actitud de cooperación y alianzas por parte } \\
\text { de la empresa (con otras empresas, con la } \\
\text { AA.PP, con el entorno) }\end{array}$ & 0.5528 & 0.5603 & 0.0453 & 121.906 \\
\hline & $\begin{array}{l}\text { La capacidad de evaluación de riesgos de las } \\
\text { inversiones }\end{array}$ & 0.5950 & 0.5917 & 0.0452 & 131.513 \\
\hline & $\begin{array}{l}\text { Los recursos financieros/estructura } \\
\text { económico financiera }\end{array}$ & 0.6118 & 0.6093 & 0.0422 & 144.866 \\
\hline & Los recursos naturales & 0.5616 & 0.5511 & 0.0463 & 121.192 \\
\hline & El tipo de proveedores & 0.6612 & 0.6628 & 0.0372 & 177.737 \\
\hline & El proceso de aprovisionamiento & 0.7460 & 0.7444 & 0.0298 & 250.656 \\
\hline & $\begin{array}{l}\text { El sistema de producción /logística / } \\
\text { almacenamiento /distribución }\end{array}$ & 0.6710 & 0.6663 & 0.0379 & 176.943 \\
\hline & El proceso de comercialización y venta & 0.6525 & 0.6552 & 0.0365 & 178.620 \\
\hline & El sistema de calidad/gestión medioambiental & 0.5829 & 0.5768 & 0.0441 & 132.209 \\
\hline & Las áreas geográficas en las que compra & 0.6207 & 0.6159 & 0.0456 & 136.133 \\
\hline & Las áreas geográficas en las que produce & 0.6334 & 0.6347 & 0.0389 & 162.742 \\
\hline & Las áreas geográficas en las que vende & 0.5122 & 0.5128 & 0.0590 & 86.870 \\
\hline \multirow[t]{5}{*}{ INNO } & $\begin{array}{l}\text { La capacidad de innovación de procesos, } \\
\text { productos o mercados }\end{array}$ & 0.7757 & 0.7773 & 0.0270 & 287.628 \\
\hline & $\begin{array}{l}\text { Las tecnologías de procesos y productos } \\
\text { utilizadas }\end{array}$ & 0.6768 & 0.6708 & 0.0384 & 176.453 \\
\hline & $\begin{array}{l}\text { El proceso de investigación/desarrollo/ } \\
\text { innovación }\end{array}$ & 0.8118 & 0.8089 & 0.0282 & 287.424 \\
\hline & El proceso de diseño & 0.7585 & 0.7574 & 0.0295 & 257.055 \\
\hline & $\begin{array}{l}\text { Capacidad de penetración en nuevos } \\
\text { mercados internacionales }\end{array}$ & 0.6314 & 0.6273 & 0.0389 & 162.195 \\
\hline
\end{tabular}

FUENTE ELABORACIÓN PROPIA. 
La significación de los diferentes elementos incluidos en el cuadro justifica la elección de los ítems como indicadores de los constructos previamente definidos. Sin embargo, el efecto de la gestión de los recursos territoriales, considerados como base de los clústeres empresariales sobre la mejora de resultados de innovación de la empresa, ha sido considerado en el largo plazo, dado que sus efectos son sobre las posibilidades de competir de las empresas. En general, no se ha consignado un efecto directo de este factor. Por ese motivo tiene interés analizar si esos recursos son vistos como generadores de innovación para las empresas de una determinada región y, en particular, generadora de innovaciones en el corto plazo.

Para evaluar dicho impacto se ha estimado la relación entre ambos constructos mediante PLS. Los resultados se recogen en el Cuadro 7. Éste muestra la validez de dicha afirmación en el caso de Vigo y su área de influencia.

En resumen, se observa que el efecto del territorio sobre la innovación es significativo en la zona de estudio. En general, los clústeres son elementos fundamentales para la constitución de los sistemas regionales de innovación, que están en la base de muchas de las políticas de innovación propuestas en los territorios. Por otra parte, la escasez de recursos en este tipo de empresas limita sus posibilidades de innovación. Además, la necesidad de financiación y de recursos físicos en la empresa es necesaria para las inversiones en innovación futura. Por ese motivo, la cooperación de la empresa aparece como una estrategia para generar redes que sirvan de base al desarrollo de mejoras en productos, procesos o servicios (Verhees y Meulenberg, 2004).

CUADRO 7 | Evaluación bootstrap del modelo estructural

\begin{tabular}{l|c|c|c|c}
\hline & $\begin{array}{c}\text { ENTIRE SAMPLE } \\
\text { ESTIMATE }\end{array}$ & $\begin{array}{c}\text { MEAN OF } \\
\text { SUBSAMPLES }\end{array}$ & STANDARD & T-STATISTIC \\
\cline { 3 - 4 } ZONE->INNO & 0.6430 & 0.6512 & 0.0262 & 245.857 \\
\hline
\end{tabular}

FUENTE ELABORACIÓN PROPIA.

En ese sentido, la gestión de los recursos territoriales es clave para la elaboración de la política de innovación de la empresa, puesto que afecta al conjunto de los elementos que la componen. Por tanto, una mejor gestión de los recursos territoriales tiene un efecto positivo sobre la innovación. De esa forma, se aceptan las teorías del milieu innovador en esa zona y la importancia que la constitución de clústeres produce para la mejora de la innovación, no sólo desde un punto de vista macroeconómico (Méndez, 2002), sino también microeconómico. 


\section{Conclusiones}

Existen muchos trabajos que relacionan territorio e innovación. La mayoría se incluyen dentro del concepto de milieu innovador siguiendo los análisis clásicos (Vázquez-Barquero, 1990). Sin embargo, es menos habitual analizar el efecto que la gestión del territorio por parte de la empresa puede tener para generar sus innovaciones. Un aspecto particular consiste en corroborar cómo los factores externos de la empresa ayudan a potenciar la innovación cuando son bien gestionados por las propias empresas.

El objetivo de este trabajo estaba orientado a determinar la relación existente entre estas dos competencias y más específicamente si la gestión de los recursos territoriales por parte de la empresa fomentaba su capacidad de innovación.

Los resultados empíricos muestran que un incremento en el uso de la gestión de los recursos territoriales produce una mejora significativa en la capacidad de innovación. Esto es coherente con otros trabajos de la literatura organizacional que insisten en la importancia de aprovechar la cercanía al mercado y al cliente dada la reducida dimensión de las empresas (Pelham, 1997). Es fácil extender esa idea a los proveedores y en general a la constitución de clústeres empresariales, confirmándose la estrecha relación entre innovación empresarial y territorio. En ese sentido, se apoya la teoría tradicional al considerar que es importante para la pyme promover la constitución de clústeres empresariales que faciliten la innovación de las empresas (Porter, 1990).

Se comprueba que la capacidad de innovación de la empresa está conformada por los procesos de I+D y de diseño para desarrollar innovaciones empresariales, junto con la necesidad de capacidad de innovación y de penetración en nuevos mercados por parte del personal de la empresa. Además, como es de esperar, el uso de la tecnología forma parte esencial de dicho proceso.

La gestión de los recursos territoriales por parte de la empresa incluye tanto la gestión de los recursos naturales disponibles en el territorio como de los recursos financieros, dado que la pyme hace uso de fuentes de financiación fundamentalmente locales. También incluye el capital relacional local, centrado en las relaciones con clientes y proveedores, al igual que los aspectos afines a ellos. Dichas relaciones son fomentadas por la capacidad de cooperación de las empresas. De modo más específico se incluye la zona donde se llevan a cabo esas relaciones, al igual que la zona de producción y el proceso asociado con ésta. Se demuestra que todos estos son elementos importantes para promover la innovación empresarial de la zona.

Como consecuencia de sus contenidos y de la relación existente, se ratifica la importancia que el capital relacional tiene para generar innovaciones en las empresas, normalmente manifestada a través de la cooperación vertical entre ellas. De igual modo, se confirma la necesidad de recursos financieros para desarrollar innovaciones. Es interesante notar cómo también los recursos naturales son fuente de innovación para las pymes regionales. Finalmente, los procesos de compra, producción y venta y los territorios asociados a esos procesos también son fuentes 
de innovación de las pymes. En general, todos ellos son elementos que permiten configurar de modo global el sistema de innovación local. IEURE

\section{Agradecimientos}

Queríamos agradecer a los evaluadores externos que han aportado ideas para la mejora de este trabajo. De igual modo agradecemos a la Fundación Provigo y al Concello de Vigo puesto que financiaron en parte dicha investigación.

\section{Referencias bibliográficas}

Acs, Z. J. \& Audretsch, D. B. (1988). Innovation in large and small firms: an empirical analysis. The American Economic Review, Vol. 78, No. 4, pp. 678-690.

Autoridad Portuaria de Vigo (31 de 12 de 2009). Memoria anual del puerto de Vigo. Recuperado el 05 de 05 de 2011, de http://www.apvigo.com/control.php?sph=a_iap=1251\%\%p_rpp=1

Balakrishnan, S. \& Fox, I. (1993). Asset Specifity, Firm Heterogeneity and Capital Structure. Strategic Management Journal, No. 14, pp. 3-16.

Benko, G. \& Lipietz, A. (2000). La richesse des régions. París: P.U.F.

Beristain, L. M. (2009). Una Revisión de la Interpretación Económica sobre la Innovación. Journal of Technology Management \& Innovation, Vol. 4, No. 4 [citado 2010-08-26], pp. 139-149.

Birley, S. \& Westhead, P. (1990). Growth and Performance Contrasts between Types of Small Firms. Strategic Management Journal, Vol. 11, No. 7, pp. 535-557.

Bontis, N. \& Fitz-Enz, J. (2002). Intellectual capital ROI: a causal map of human capital antecedents and consequents. Journal of Intellectual Capital, Vol. 3, No. 3, pp. 223-247.

Cal, M.; Verdugo, M. \& Jardón, C. (2007). Internacionalización-cooperacion nas pemes de Vigo e a sua area metropolitana. Revista galega de Economia, Vol. 16, No. 1, pp. 173-186.

Capo-Vicedo, J.; Exposito-Langa, M. \& Masia-Buades, E. (2007). La importancia de los clusters para la competitividad de las PYME en una economía global. EURE, Vol. 33, No. 98, pp. 119-133.

Chin, W. (1998). The partial Least squares approach to structural equation model. En G. Marcoulides (Ed.). Modern methods for business Research. Mahwah, New Jersey, London: Lawrence Erlbaum associates publisher.

CHUVI (12 de 12 de 2009). Complejo Hospitalario Universitario de Vigo. Recuperado el 08 de 05 de 2011, de Investigación: http://chuvi.sergas.es/ga/fundacionbiomedica/actividade/Paginas/proxectos_activos.aspx

Cooke, P. \& Morgan, K. (1998). The associational economy: firms, regions and innovation. Oxford: Oxford University Press.

Cronbach, L. J. (1951). Coefficient alpha and the internal structure of tests. Psychometrika, Vol. 16, No. 3, pp. 297-334.

De Brentani, U. \& Kleinschmidt, E. (2004). Corporate Culture and Commitment: Impact on Performance of International New Product Development Programs. Journal of Product Innovation Management, Vol. 21, No. 5, pp. 309-33.

Deshpande, S. \& Golhar, D. (1994). "HRM Practice in Large and Small Manufacturing Firms: A Comparative Study". Journal of Small Business Management, Vol. 32, No. 2, pp. 49-56. 
Drucker, P. F. (1988). La innovación y el empresariado innovador: la práctica y los principios. México: Hermes.

Efron, B. (1979). Bootstrap methods: another look at the jackknife. Annals of Statistics, No. 7, pp. 1-26. Entrialgo, M.; Fernández, E. \& Vázquez, C. (2001). El efecto de las características de la organización en el comportamiento emprendedor. Revista Europea de Dirección y Economía de la Empresa, Vol. 10, No. 3, pp. 25-40.

Feldman, M. (1994). The geography of innovation. Dordrecht: Kluwer.

Friedman, J. (1992). Empowerment: the politics of alternative development. Oxford: Blackwell.

Fukugawa, N. (2006). Determining factors in innovation of small firm networks: A case of cross industry groups in Japan. Small Business Economics, Vol. 27, No. 2, pp. 181-193.

González, J.; Figueroa, P.; Fernández-Jardón, C. \& Pita, R. (2001). Visión estratégica del sistema productivo de Vigo y su área de influencia. Vigo: Instituto de desarrollo Caixanova.

González, J.; Figueroa, P.; González, M.; Jardón, C. \& Martos, S. (2006). Visión estratégica del sistema empresarial del área Metropolitana de Vigo. Evolución 2000-2005 y expectativas. Vigo: Instituto de desarrollo Caixanova.

Grant, R. (2005). Contemporary Strategy Analysis. Oxford: Blackwell.

Hagerstrand, T. (1953). Diffusion of Innovations. Chicago: Allan Pred.

Hair, J. F.; Black, W.; Babin, B. \& Anderson, R. E. (2006). Multivariate data analysis. Upper Saddle River, NJ: Pearson / Prentice Hall.

Helfat, C. (1994). Firm-Specifity in Corporate Applied R \& D. Organization Science, Vol. 5, No. 2, pp. 173-184.

Hii, J. \& Neely, N. (2000). Innovative capacity of firms: on why some firms are more innovative than others. 7th International Annual EurOMA Conference. Ghent.

I.G.E. (2009). Datos estadísticos. Recuperado el 04 de 05 de 2011, de Insitituto galego de Estatística: http://www.ige.eu

IEO (11 de 11 de 2009). Centro de Vigo del Instituto Oceanográfico Español. Recuperado el 05 de 05 de 2011, de http://www.vi.ieo.es/general/principal.aspx ?web=covigo.aspx

IIM (31 de 12 de 2009). Instituto de investigaciones marinas. Recuperado el 05 de 05 de 2011, de Memoria de investigación 2009: http://iim.csic.es/Documentos/memoria2009.pdf

Ireland, R. D. \& Webba, J. W. (2007). Strategic entrepreneurship: Creating competitive advantage through streams of innovation. Business Horizons, Vol. 50, No. 1, pp. $49-59$.

Jardón, C. \& Martos, M. (2010). Determinación de Competencias Distintivas en Pymes: El Caso de Vigo y su Área de Influencia (España). Visión de Futuro, Vol. 14, No. 2, pp. 1-15.

Kim, D. J. \& Kogut, B. (1996). Technological Platforms and Diversification. Organization Science, Vol. 7, No. 3, pp. 283-301.

Klofsten, M. \& Scheele, J. (2003). Innovation and Small Business in the Third World. International Small Business Journal, Vol. 21, No. 3, pp. 354- 357.

Lloyd-Reason, L.; Muller, K. \& Wall, S. (2002). Innovation and Educational Policy in SMEs: A Czech Perspective. Education \& Training, Vol. 44, No. 8-9, pp. 378-387.

Maillat, D. \& Grosjean, N. (1999). Globalisation et systèmes territoriaux de production. Neuchâtel: Working Paper IRER 9906a, Université de Neuchâtel.

Malhotra, N. (1981). A scale to measure self concepts and product concepts. Journal of Marketing Research, Vol. 18, November, pp. 456-464. 
McCann, P. \& Arit, P. (2006). Clusters and regional development: Some cautionary observations from the semiconductor industry. Information Economics and Policy, No. 18, pp. 157-180.

McLaran, P. \& McGowan, P. (1999). Managing Service Quality For Competitive Advantage in Small Engineering. Rms. International Journal of Entrepreneurial Behaviour and Research, Vol. 5 , No. 2, pp. 35-47.

Méndez, R. (2002). Innovación y desarrollo territorial: algunos debates teóricos recientes. EURE, Vol. 28, No. 84, pp. 63-83.

Narver, J. \& Slater, S. (1990). The Effect of a Market Orientation on Business Profitability. Journal of Marketing, Vol. 54, No. 4 (October), pp. 20-35.

Newbold, P.; Carlson, W. \& Thorne, B. (2002). Statistics for Business and Economics. NY: Prentice Hall. Nunnelly, J. C. (1978). Psychometric Theory. New York: McGraw Hill.

Papaconstantinou, G. (1997). Technology and industrial performance. The OECD Observer, No. 204, Feb/Mar, pp. 6-10.

Pavitt, K. (1984). Sectorial Patterns of Technical Change: Towards a Taxonomy and a Theory. Research Policy, Vol. 13, No. 6, pp. 343-373.

Pelham, A. (1997). Mediating Influences on the Relationship Between Market Orientation and Profitability in Small Industrial Firms. Journal of Marketing Theory and Practice, Vol. 5, No. 3 (Summer), pp. 55-76.

Pérez, B. \& Carrillo, E. (2000). Desarrollo local: manual de uso. Madrid: ESIC- Federación Andaluza de Municipios y Provincias.

Porter, M. (1985). Competitive advantage. New York: The Free Press.

Porter, M. (1990). The Competitive Advantage of Nations. New York: The Free Press.

Provigo (2002). Fundación Provigo. Recuperado el 07 de 09 de 2010, de http://www.fundacionprovigo.org/adjuntos/cTexto/12/planestrategico2010.pdf

Retgalia (1 de 1 de 2010). Red de centros tecnológicos de Galicia. Recuperado el 08 de 05 de 2011, de http://www.retgalia.org/centros.html

Ricardo, D. (1817). On the Principles of Political Economy and Taxation. London: John Murray.

Rodeiro, D. \& López, M. ( 2007 ). A innovación como factor clave na competitividade empresarial: un estudo empírico en pemes galega. Revista galega de economia, Vol. 16, No. 2, pp. 5-22.

Rosenberg, N. (2000). Schumpeter and the endogeneity of technology. London: Routledge.

Schumpeter, J. (1934). The Theory of Economic Development. Cambridge, MA: Harvard University Press.

Sforzi, F. (1999). La teoría marshalliana para explicar el desarrollo local. En F. Rodríguez (Ed.). Manual de desarrollo local. Oviedo: Trea Ediciones.

Teach, R. (1990). Entrepreneurship and Marketing: The Pricing and Distribution Link To Success. En G. Hills, R. Laforge, \& H. Welsch (Eds.). Research at the Marketing/Entrepreneurship Interface. Chicago: The University of Illinois at Chicago.

Teece, D. J. (2000). Managing intellectual capital. New York: Oxford University Press.

UVigo. (03 de 12 de 2009). Universidad de Vigo/ investigación. Recuperado el 05 de 05 de 2011, de Memoria del sexenio 2003-2008: http://www.uvigo.es/opencms/export/sites/uvigo/sites/default/uvigo/DOCUMENTOS/investigacion/Sexenio_de_investigacixn_2003-2008.pdf

Vázquez-Barquero, A. (1999). Inward Investment and Endogenous Development. The Convergence of the Strategies of Large Firms and Territories? Entrepreneurship \& Regional Development, Vol. 11, pp. 79-93. 
Vázquez-Barquero, A. (1990). Conceptualizing Regional Dynamics in Recently Industrialyzed Countries. Environment and Planning, Vol. 22, pp. 477-491.

Vázquez-Barquero, A. (1999). Dinámica productiva y desarrollo urbano: La respuesta de la ciudad de Vitoria (País Vasco) a los desafíos de la globalización. EURE, Vol. 25, No.74, pp. 19-33.

Verhees, F. \& Meulenberg, M. (2004). Market Orientation, Innovativeness, Product Innovation, and Performance in Small Firms. Journal of Small Business Management, Vol. 42, No. 2, pp. 86-91.

Wagner, S. (2006). A firm's responses to deficient suppliers and competitive advantage. Journal of Business Research, Vol. 59, pp. 686-695.

Zahra, S. A. \& Das, S. R. (1993). Building Competitive Advantage on Manufacturing Resources. Long Range Planning, Vol. 26, No. 2, pp. 90-97. 